\title{
Bricklayer Attack: A Side-Channel Analysis on the ChaCha Quarter Round
}

Alexandre Adomnicai
${ }^{1,3}$ Jacques J.A. Fournier ${ }^{2}$ Laurent Masson ${ }^{1}$
${ }^{1}$ Trusted Objects
${ }^{2}$ CEA-Leti
${ }^{3}$ EMSE

INDOCRYPT 2017

Chennai, December 13th

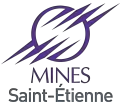




\section{Side-Channel Attacks}

$\triangleright$ Cryptographic primitives are designed to be finally executed on a physical system.

$\triangleright$ The physical characteristics of the computing platform produce side effects depending on the processed data

- Power consumption $\circ$ Electromagnetic emanations $\circ$ Time execution $\circ$ Sound ...

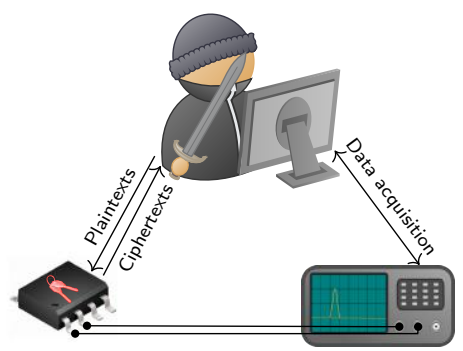




\section{Side-Channel Attacks}

$\triangleright$ Cryptographic primitives are designed to be finally executed on a physical system.

$\triangleright$ The physical characteristics of the computing platform produce side effects depending on the processed data

- Power consumption $\circ$ Electromagnetic emanations $\circ$ Time execution $\circ$ Sound ...

$\triangleright$ One can measure these side effects to get information on the processed values during sensitive operations

$\triangleright$ Using an appropriate leakage model, one can recover the secrets involved in calculations

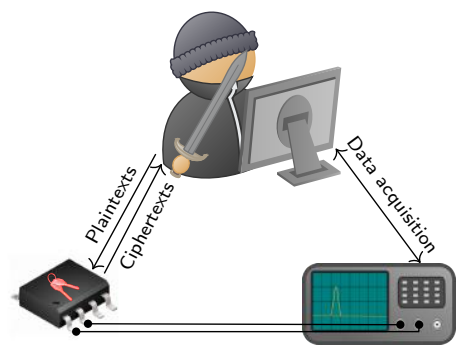




\section{Selection Functions}

$\triangleright$ Differential Power/Electromagnetic analyses target an intermediate state $y$ which depends on a known input $x$ and a secret $k$.

$\triangleright$ This value is defined by a selection function $\varphi(x, k)=y$. 


\section{Selection Functions}

$\triangleright$ Differential Power/Electromagnetic analyses target an intermediate state $y$ which depends on a known input $x$ and a secret $k$.

$\triangleright$ This value is defined by a selection function $\varphi(x, k)=y$.

$\triangleright$ High non-linearity is a valuable property as it ensures a good distinguishability between correct and incorrect key guesses.

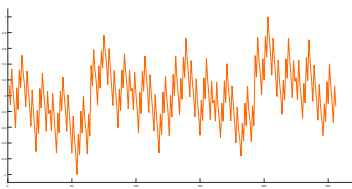

$\varphi(x, k)=x \oplus k$

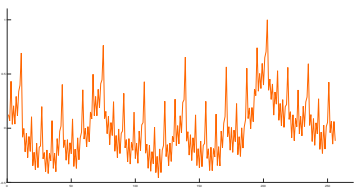

$\varphi(x, k)=x \boxplus k$

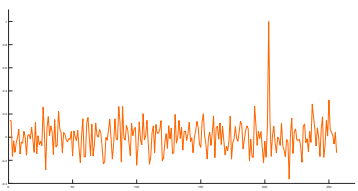

$\varphi(x, k)=\operatorname{AES}_{\text {Sbox }}(x \oplus k)$

Simulation of Correlation Power Analyses (CPA) in the Hamming Weight model 


\section{The ChaCha Family of Stream Ciphers}

$\triangleright$ ChaCha is a family of stream ciphers introduced by Daniel J. Bernstein in 2008.

$\triangleright$ ChaCha is based on Salsa20 (eSTREAM portfolio) while improving diffusion without performance hit. 


\section{The ChaCha Family of Stream Ciphers}

$\triangleright$ ChaCha is a family of stream ciphers introduced by Daniel J. Bernstein in 2008.

$\triangleright$ ChaCha is based on Salsa20 (eSTREAM portfolio) while improving diffusion without performance hit.

$\triangleright$ ChaCha20 has been widely adopted in practice

- Android phones (ChaCha20-Poly1305 AEAD used in TLS with Chrome)

- Apple HomeKit for loT devices (ChaCha20-Poly1305 AEAD with HKDF-SHA-512 derived keys)

- Linux kernel $4.8+$ (/dev/urandom based on ChaCha20)

- OpenBSD (ChaCha20 now replaces RC4 for pseudo-random number generator)

- Numerous security protocols (TLS, SSH, IPsec, ...) 


\section{How to Dance the ChaCha}

$\triangleright$ Operate like an iterative 512-bit block cipher using CTR mode 


\section{How to Dance the ChaCha}

$\triangleright$ Operate like an iterative 512-bit block cipher using CTR mode

$\triangleright$ The internal state consists in a $4 \times 4$ matrix of 32 -bit elements

\begin{tabular}{cccc} 
'expa' 'nd 3' '2-by' 'te k' \\
$k_{0}$ & $k_{1}$ & $k_{2}$ & $k_{3}$ \\
$k_{4}$ & $k_{5}$ & $k_{6}$ & $k_{7}$ \\
nonce $_{0}$ & nonce & nonce & nonce $_{3}$ \\
\multicolumn{4}{c}{ Initial State }
\end{tabular}




\section{How to Dance the ChaCha}

$\triangleright$ Operate like an iterative 512-bit block cipher using CTR mode

$\triangleright$ The internal state consists in a $4 \times 4$ matrix of 32 -bit elements

$\triangleright$ Every round is divided in quarter rounds (QR)

$\begin{array}{cccc}\text { 'expa' 'nd 3' '2-by' 'te k' } \\ k_{0} & k_{1} & k_{2} & k_{3} \\ k_{4} & k_{5} & k_{6} & k_{7} \\ \text { nonce }_{0} & \text { nonce } & \text { nonce } & \text { nonce } \\ & \text { Initial State }\end{array}$




\section{How to Dance the ChaCha}

$\triangleright$ Operate like an iterative 512-bit block cipher using CTR mode

$\triangleright$ The internal state consists in a $4 \times 4$ matrix of 32 -bit elements

$\triangleright$ Every round is divided in quarter rounds (QR)

$\triangleright$ QRs only use Additions, Rotations and XORs: ARX-based cipher

$\begin{array}{cccc}\text { 'expa' 'nd 3' '2-by' 'te k' } \\ k_{0} & k_{1} & k_{2} & k_{3} \\ k_{4} & k_{5} & k_{6} & k_{7} \\ \text { nonce } & \text { nonce } 1 & \text { nonce } & \text { nonce } 3 \\ & \text { Initial State }\end{array}$

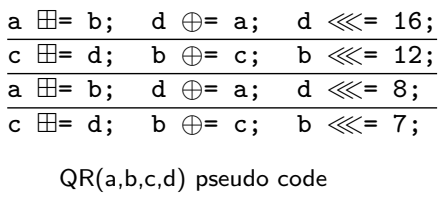




\section{How to Dance the ChaCha}

$\triangleright$ Operate like an iterative 512-bit block cipher using CTR mode

$\triangleright$ The internal state consists in a $4 \times 4$ matrix of 32 -bit elements

$\triangleright$ Every round is divided in quarter rounds (QR)

$\triangleright$ QRs only use Additions, Rotations and XORs: ARX-based cipher

$\triangleright$ If the round number is odd/even QRs are applied on columns/diagonals

$\begin{array}{cccc}\text { 'expa' 'nd 3' '2-by' 'te k' } & k_{2} & k_{3} \\ k_{0} & k_{1} & k_{2} & k_{7} \\ k_{4} & k_{5} & k_{6} & \text { nonce }_{3} \\ \text { nonce }_{0} & \text { nonce } & \text { nonce } & \text { nonce } \\ & \text { Initial State }\end{array}$

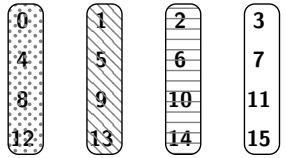

(a) Even round

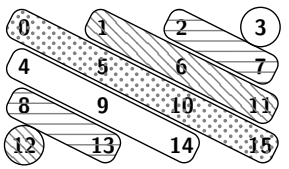

(b) Odd round 


\section{How to Dance the ChaCha}

$\triangleright$ Operate like an iterative 512-bit block cipher using CTR mode

$\triangleright$ The internal state consists in a $4 \times 4$ matrix of 32 -bit elements

$\triangleright$ Every round is divided in quarter rounds (QR)

$\triangleright$ QRs only use Additions, Rotations and XORs: ARX-based cipher

$\triangleright$ If the round number is odd/even QRs are applied on columns/diagonals

$\triangleright$ After the last round, the keystream is obtained by adding the current state with the initial one

$\begin{array}{cccc}\text { 'expa' 'nd 3' '2-by' 'te k' } \\ k_{0} & k_{1} & k_{2} & k_{3} \\ k_{4} & k_{5} & k_{6} & k_{7} \\ \text { nonce }_{0} & \text { nonce } 1 & \text { nonce } & \text { nonce } \\ & \text { Initial State }\end{array}$

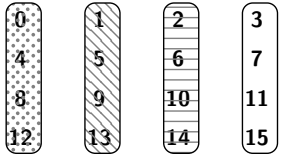

(a) Even round

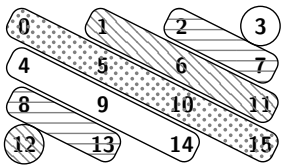

(b) Odd round

\begin{tabular}{lll}
$\mathrm{a} \boxplus=\mathrm{b} ;$ & $\mathrm{d} \oplus=\mathrm{a} ;$ & $\mathrm{d} \lll=16 ;$ \\
\hline $\mathrm{c} \boxplus=\mathrm{d} ;$ & $\mathrm{b} \oplus=\mathrm{c} ;$ & $\mathrm{b} \lll=12 ;$ \\
\hline $\mathrm{a} \boxplus=\mathrm{b} ;$ & $\mathrm{d} \oplus=\mathrm{a} ;$ & $\mathrm{d} \lll=8 ;$ \\
\hline $\mathrm{c} \boxplus=\mathrm{d} ;$ & $\mathrm{b} \oplus=\mathrm{c} ;$ & $\mathrm{b} \lll=7 ;$
\end{tabular}

$\mathrm{QR}(\mathrm{a}, \mathrm{b}, \mathrm{c}, \mathrm{d})$ pseudo code 


\section{Attack published at DATE 2017}

$\triangleright$ All key words are directly involved during the first column round.

$\begin{array}{cccc}\operatorname{expa} & \text { 'nd 3' '2-by' 'te k' } \\ k_{0} & k_{1} & k_{2} & k_{3} \\ k_{4} & k_{5} & k_{6} & k_{7} \\ \text { nonce }_{0} & \text { nonce }_{1} & \text { nonce }_{2} & \text { nonce }_{3}\end{array}$




\section{Attack published at DATE 2017}

$\triangleright$ All key words are directly involved during the first column round.

$\triangleright$ They interact with the only changing variable: the nonce.

$\begin{array}{cccc}\text { 'expa' 'nd 3' '2-by' 'te k' } \\ k_{0} & k_{1} & k_{2} & k_{3} \\ k_{4} & k_{5} & k_{6} & k_{7} \\ \text { nonce }_{0} & \text { nonce }_{1} & \text { nonce }_{2} & \text { nonce }_{3}\end{array}$

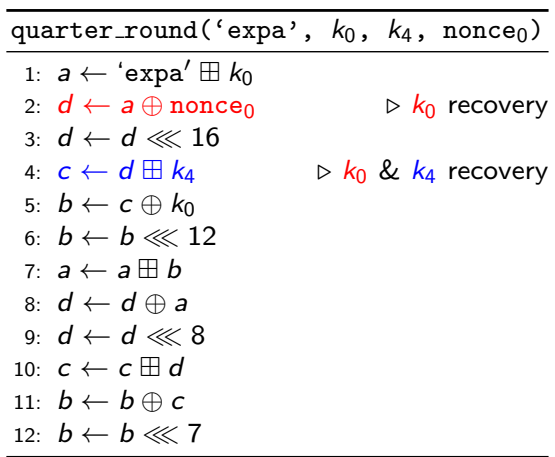




\section{Attack published at DATE 2017}

$\triangleright$ All key words are directly involved during the first column round.

$\triangleright$ They interact with the only changing variable: the nonce.

$\triangleright$ The entire key can be recovered using power/electromagnetic analyses [2].

$\begin{array}{cccc}\text { 'expa' 'nd 3' '2-by' 'te k' } \\ k_{0} & k_{1} & k_{2} & k_{3} \\ k_{4} & k_{5} & k_{6} & k_{7} \\ \text { nonce }_{0} & \text { nonce }_{1} & \text { nonce }_{2} & \text { nonce }_{3}\end{array}$

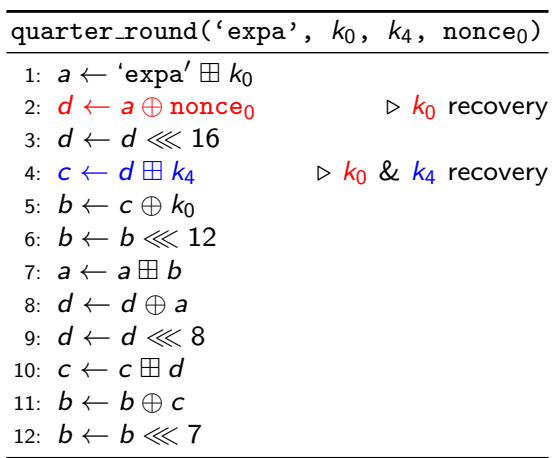




\section{Attack published at DATE 2017}

$\triangleright$ All key words are directly involved during the first column round.

$\triangleright$ They interact with the only changing variable: the nonce.

$\triangleright$ The entire key can be recovered using power/electromagnetic analyses [2].

$\triangleright k_{0,1,2,3}$ are retrieved using $\varphi(x, k)=x \oplus k$

$\begin{array}{cccc}\text { 'expa' 'nd 3' '2-by' 'te k' } \\ k_{0} & k_{1} & k_{2} & k_{3} \\ k_{4} & k_{5} & k_{6} & k_{7} \\ \text { nonce }_{0} & \text { nonce }_{1} & \text { nonce }_{2} & \text { nonce }_{3}\end{array}$

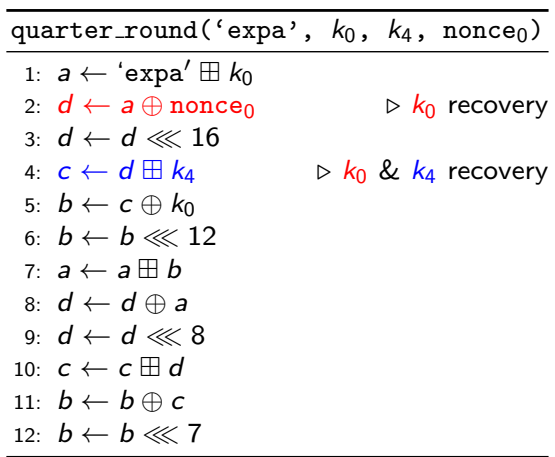




\section{Attack published at DATE 2017}

$\triangleright$ All key words are directly involved during the first column round.

$\triangleright$ They interact with the only changing variable: the nonce.

$\triangleright$ The entire key can be recovered using power/electromagnetic analyses [2].

$\triangleright k_{0,1,2,3}$ are retrieved using $\varphi(x, k)=x \oplus k$

$\triangleright k_{4,5,6,7}$ are retrieved using $\varphi(x, k)=x \boxplus k$

$\begin{array}{cccc}\text { 'expa' 'nd 3' '2-by' 'te k' } \\ k_{0} & k_{1} & k_{2} & k_{3} \\ k_{4} & k_{5} & k_{6} & k_{7} \\ \text { nonce }_{0} & \text { nonce }_{1} & \text { nonce }_{2} & \text { nonce }_{3}\end{array}$

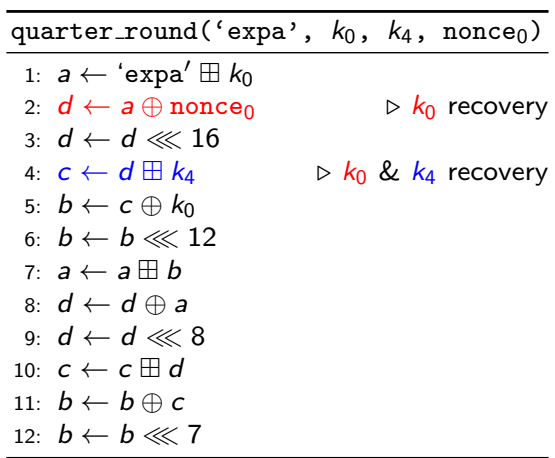




\section{Practical experiments}

$\triangle$ All practical experiments were done on an ARM Cortex-M3 clocked at $24 \mathrm{MHz}$ using

- Langer HF-U 5 near-field probe $(30 \mathrm{MHz}-3 \mathrm{GHz})$

Langer PA 303 BNC preamplifier (+ 30dB)

- LeCroy WaveSurfer 10 oscilloscope (10GS/s)

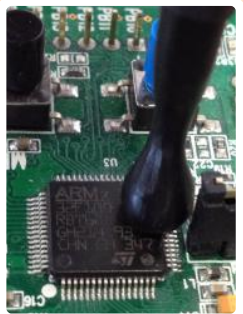

Device Under Test 


\section{Practical experiments}

$\triangle$ All practical experiments were done on an ARM Cortex-M3 clocked at $24 \mathrm{MHz}$ using

- Langer HF-U 5 near-field probe $(30 \mathrm{MHz}-3 \mathrm{GHz})$

Langer PA 303 BNC preamplifier (+30dB)

- LeCroy WaveSurfer 10 oscilloscope (10GS/s)

$\triangleright$ Application of both attacks on two different ChaCha20 implementations

- OpenSSL (1.0.1f) compiled using the GNU ARM C compiler (5.06)

- Homemade ARM assembly

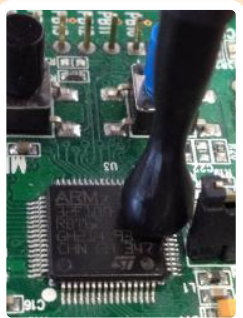

Device Under Test

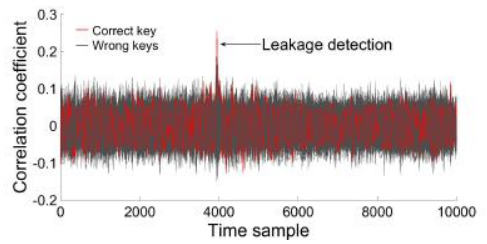

(a) C compiled

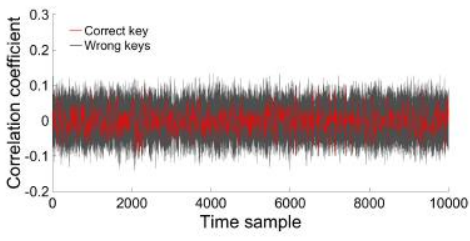

(b) ARM Assembly 


\section{Assembly VS C compiled}

\section{-00 Compilation}

LDR
LDR
ADD
STR
LDR
LDR
EORS
LSLS
LDR
LDR
EORS
ORR
STR
LDR
LDR
ADD
STR
LDR
LDR
EORS
LSLS
LDR
LDR
EORS
ORR
STR

\section{-03 Compilation}

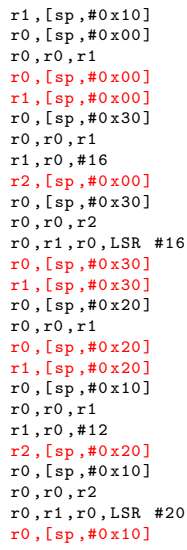

$\mathrm{r} 1,[\mathrm{sp}, \# 0 \times 10]$

$\mathrm{ro},[\mathrm{sp}, \# 0 \times 00]$

$r 0, r 0, r 1$

$\mathrm{ro},[\mathrm{sp}, \# 0 \mathrm{x} 00]$

$\mathrm{r} 1,[\mathrm{sp}, \# 0 \times 00]$

$\mathrm{ro},[\mathrm{sp}, \# 0 \times 30]$

$\mathrm{r} 0, \mathrm{r} 0, \mathrm{r} 1$

ro, ro, \#16

ro, [sp, \#0 x 30]

$\mathrm{r} 1,[\mathrm{sp}, \# 0 \times 30]$

$\mathrm{ro},[\mathrm{sp}, \# 0 \times 20]$

ro, ro, r 1

ro, [sp, \#0 x 20]

$\mathrm{r} 1,[\mathrm{sp}, \# 0 \times 20]$

$\mathrm{ro},[\mathrm{sp}, \# 0 \times 10]$

$\mathrm{r} 0, \mathrm{r} 0, \mathrm{r} 1$

ro, ro, \#20

$\mathrm{ro},[\mathrm{sp}, \# 0 \times 10]$

\section{ARM Assembly}

LDR

LDR

LDR

LDR

$A D D$

EOR

ROR

ADD

EOR

ROR

ADD

STR

EOR

ROR

STR

ADD

STR

EOR

ROR

STR

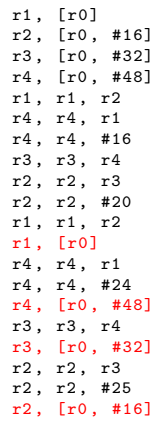




\section{Information Leakage \& Implementation Aspects}

$\triangleright$ Load/store architectures divide instructions into 2 categories

- Memory accesses

- Arithmetic Logic Unit (ALU) operations
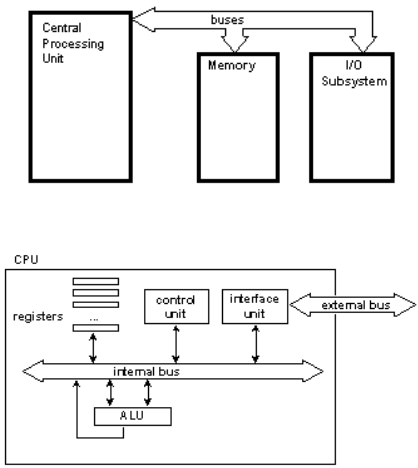


\section{Information Leakage \& Implementation Aspects}

$\triangleright$ Load/store architectures divide instructions into 2 categories

- Memory accesses

- Arithmetic Logic Unit (ALU) operations

$\triangleright$ When a CPU loads values from RAM to registers

- The memory address is placed on the address bus

- The data contained at the address is moved to the data bus

- The data is transferred into a register
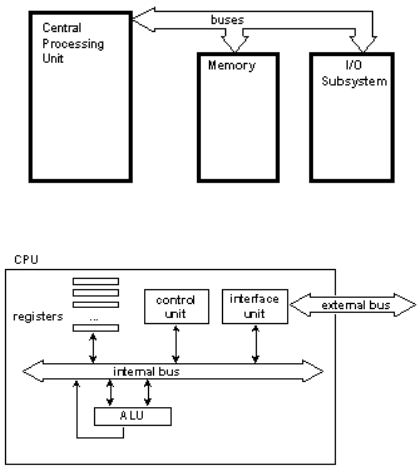


\section{Information Leakage \& Implementation Aspects}

$\triangleright$ Load/store architectures divide instructions into 2 categories

- Memory accesses

- Arithmetic Logic Unit (ALU) operations

$\triangleright$ When a CPU loads values from RAM to registers

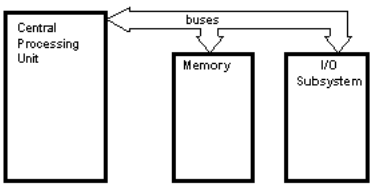

- The memory address is placed on the address bus

- The data contained at the address is moved to the data bus

- The data is transferred into a register

$\triangleright$ When a CPU performs ALU operations

- The operand registers' content are transferred to the ALU

- The ALU performs the calculation and places the result in the output register

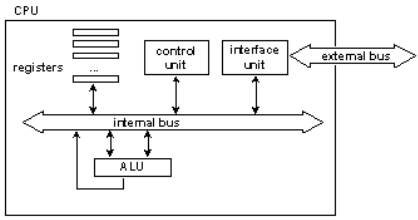

Is it easier to exploit leakages in relation to memory instructions? 


\section{Focusing on Memory Instructions}

$\triangleright$ Focusing on memory accesses imply to analyze the whole QR 


\section{Focusing on Memory Instructions}

$\triangleright$ Focusing on memory accesses imply to analyze the whole QR

$\triangleright$ The simplest selection function is defined by focusing the first STR instruction

$$
\varphi_{1}\left(\text { nonce }_{i}, k_{i} \| k_{i+4}\right)=\text { nonce }_{i} \oplus \tilde{k}_{i} \lll 16 \boxplus k_{i+4} \oplus k_{i} \lll 12 \boxplus \tilde{k}_{i}
$$

where $\tilde{k}_{i}=k_{i} \boxplus$ constant $_{i}$ 


\section{Focusing on Memory Instructions}

$\triangleright$ Focusing on memory accesses imply to analyze the whole QR

$\triangleright$ The simplest selection function is defined by focusing the first STR instruction

$$
\varphi_{1}\left(\text { nonce }_{i}, k_{i} \| k_{i+4}\right)=\text { nonce }_{i} \oplus \tilde{k}_{i} \lll 16 \boxplus k_{i+4} \oplus k_{i} \lll 12 \boxplus \tilde{k}_{i}
$$

where $\tilde{k}_{i}=k_{i} \boxplus$ constant $_{i}$

$\triangleright \varphi_{1}$ implies a side-channel attack on 2 key words at once (i.e. $\left.|\mathcal{K}|=2^{64}\right) \Rightarrow$ undoable in practice! 


\section{Divide \& Conquer}

$\triangleright$ It has been proved there is still a correlation when predicting a subpart of the word [4] 


\section{Divide \& Conquer}

$\triangleright$ It has been proved there is still a correlation when predicting a subpart of the word [4]

$\triangleright$ Targeting $n$ bits of $y=\varphi_{1}$ (nonce $\left._{i}, k_{i} \| k_{i+4}\right)$ does not lead to a complexity equal to $2^{2 n}$ 


\section{Divide \& Conquer}

$\triangleright$ It has been proved there is still a correlation when predicting a subpart of the word [4]

$\triangleright$ Targeting $n$ bits of $y=\varphi_{1}$ (nonce $\left._{i}, k_{i} \| k_{i+4}\right)$ does not lead to a complexity equal to $2^{2 n}$

$\triangleright$ The key search space depends on the windows' size $n$

$$
|\mathcal{K}|= \begin{cases}2^{4 n}, & \text { if } n \leq 4 \\ 2^{3 n+4}, & \text { if } 4 \leq n \leq 12 \\ 2^{2 n+16}, & \text { if } 13 \leq n \leq 16 \\ 2^{n+32}, & \text { otherwise }\end{cases}
$$

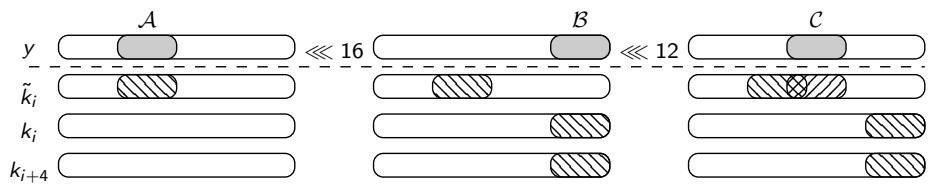

D\&C approach on the ChaCha QR, $n=8$ 


\section{Divide \& Conquer}

$\triangleright$ It has been proved there is still a correlation when predicting a subpart of the word [4]

$\triangleright$ Targeting $n$ bits of $y=\varphi_{1}$ (nonce $_{i}, k_{i} \| k_{i+4}$ ) does not lead to a complexity equal to $2^{2 n}$

$\triangleright$ The key search space depends on the windows' size $n$

$$
|\mathcal{K}|= \begin{cases}2^{4 n}, & \text { if } n \leq 4 \\ 2^{3 n+4}, & \text { if } 4 \leq n \leq 12 \\ 2^{2 n+16}, & \text { if } 13 \leq n \leq 16 \\ 2^{n+32}, & \text { otherwise }\end{cases}
$$

$\triangleright \varphi_{2, n}\left(\right.$ nonce $\left._{i}, \tilde{k}_{i}^{\mathcal{A}}\left\|k_{i}^{\mathcal{B}}\right\| k_{i+4}^{\mathcal{B}} \| \tilde{k}_{i}^{\mathcal{C}}\right)=$ nonce $_{i}^{\mathcal{A}} \oplus \tilde{k}_{i}^{\mathcal{A}} \boxplus_{n} k_{i+4}^{\mathcal{B}} \oplus k_{i}^{\mathcal{B}} \boxplus_{n} \tilde{k}_{i}^{\mathcal{C}}$

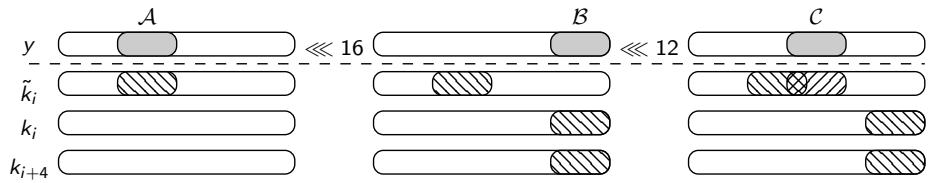

D\&C approach on the ChaCha QR, $n=8$ 


\section{Focusing on the QR}

$\triangleright$ We performed software simulations using the Hamming Weight model (without any additional noise) and random nonces 


\section{Focusing on the $Q R$}

$\triangleright$ We performed software simulations using the Hamming Weight model (without any additional noise) and random nonces

$\triangleright$ As expected, the right key matches with the highest coefficient but others too $\Rightarrow$ collisions! 


\section{Focusing on the $Q R$}

$\triangleright$ We performed software simulations using the Hamming Weight model (without any additional noise) and random nonces

$\triangleright$ As expected, the right key matches with the highest coefficient but others too $\Rightarrow$ collisions!

\section{Proposition}

An attack on $\varphi_{2, n}$ returns up to $n \cdot 2^{n+2}$ collisions.

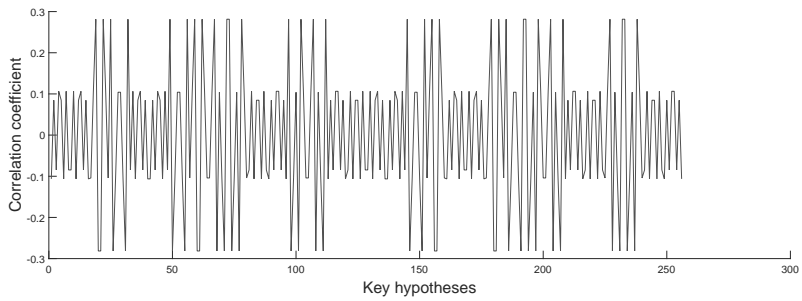




\section{Focusing on the QR}

$\triangleright$ On top of collisions, $\varphi_{2, n}$ is a victim of carry propagations

$\varphi_{2, n}\left(\right.$ nonce $\left._{i}, \tilde{k}_{i}^{\mathcal{A}}\left\|k_{i}^{\mathcal{B}}\right\| k_{i+4}^{\mathcal{B}} \| \tilde{k}_{i}^{\mathcal{C}}\right)=$ nonce $_{i}^{\mathcal{A}} \oplus \tilde{k}_{i}^{\mathcal{A}} \boxplus_{n} k_{i+4}^{\mathcal{B}} \oplus k_{i}^{\mathcal{B}} \boxplus_{n} \tilde{k}_{i}^{\mathcal{C}}$

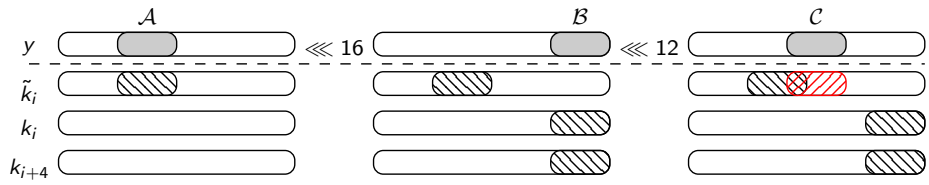

D\&C approach on the ChaCha QR, $n=8$ 


\section{Focusing on the QR}

$\triangleright$ On top of collisions, $\varphi_{2, n}$ is a victim of carry propagations

$\triangleright$ The attack should be run twice: with and without taking the carry into consideration

$$
\varphi_{2, n}\left(\text { nonce }_{i}, \tilde{k}_{i}^{\mathcal{A}}\left\|k_{i}^{\mathcal{B}}\right\| k_{i+4}^{\mathcal{B}} \| \tilde{k}_{i}^{\mathcal{C}}\right)=\text { nonce }_{i}^{\mathcal{A}} \oplus \tilde{k}_{i}^{\mathcal{A}} \boxplus_{n} k_{i+4}^{\mathcal{B}} \oplus k_{i}^{\mathcal{B}} \boxplus_{n} \tilde{k}_{i}^{\mathcal{C}}
$$

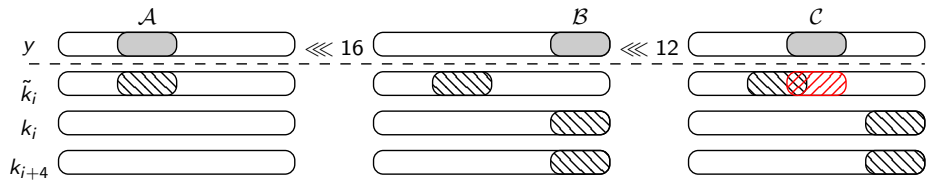

D\&C approach on the ChaCha QR, $n=8$ 


\section{Benefits of the Inverse Quarter Round}

\begin{tabular}{lll}
$\mathrm{a} \boxplus=\mathrm{b} ;$ & $\mathrm{d} \oplus=\mathrm{a} ;$ & $\mathrm{d} \lll=16 ;$ \\
\hline $\mathrm{c} \boxplus=\mathrm{d} ;$ & $\mathrm{b} \oplus=\mathrm{c} ;$ & $\mathrm{b} \lll=12 ;$ \\
$\mathrm{a} \boxplus=\mathrm{b} ;$ & $\mathrm{d} \oplus=\mathrm{a} ;$ & $\mathrm{d} \lll=8 ;$ \\
$\mathrm{c} \boxplus=\mathrm{d} ;$ & $\mathrm{b} \oplus=\mathrm{c} ;$ & $\mathrm{b} \lll=7 ;$
\end{tabular}

QR(a,b,c,d) pseudo code

\begin{tabular}{lll}
$\mathrm{b} \ggg=7 ;$ & $\mathrm{b} \oplus=\mathrm{c} ;$ & $\mathrm{c} \boxminus=\mathrm{d} ;$ \\
\hline $\mathrm{d} \ggg=8 ;$ & $\mathrm{d} \oplus=\mathrm{a} ;$ & $\mathrm{a} \boxminus=\mathrm{b} ;$ \\
$\mathrm{b} \ggg=12 ;$ & $\mathrm{b} \oplus=\mathrm{c} ;$ & $\mathrm{c} \boxminus=\mathrm{d} ;$ \\
\hline $\mathrm{d} \ggg=16 ;$ & $\mathrm{d} \oplus=\mathrm{a} ;$ & $\mathrm{a} \boxminus=\mathrm{b} ;$
\end{tabular}

$\operatorname{IQR}(\mathrm{a}, \mathrm{b}, \mathrm{c}, \mathrm{d})$ pseudo code 


\section{Benefits of the Inverse Quarter Round}

a $\boxplus=b ; \quad d \oplus=a ; \quad d \lll=16 ;$

\begin{tabular}{lll}
\hline $\mathrm{c} \boxplus=\mathrm{d} ;$ & $\mathrm{b} \oplus=\mathrm{c} ;$ & $\mathrm{b} \lll=12 ;$ \\
$\mathrm{a} \boxplus=\mathrm{b} ;$ & $\mathrm{d} \oplus=\mathrm{a} ;$ & $\mathrm{d} \lll=8 ;$ \\
$\mathrm{c} \boxplus=\mathrm{d} ;$ & $\mathrm{b} \oplus=\mathrm{c} ;$ & $\mathrm{b} \lll=7 ;$
\end{tabular}

QR(a,b,c,d) pseudo code

\begin{tabular}{lll}
$\mathrm{b} \ggg=7 ;$ & $\mathrm{b} \oplus=\mathrm{c} ;$ & $\mathrm{c} \boxminus=\mathrm{d} ;$ \\
\hline $\mathrm{d} \ggg=8 ;$ & $\mathrm{d} \oplus=\mathrm{a} ;$ & $\mathrm{a} \boxminus=\mathrm{b} ;$ \\
$\mathrm{b} \ggg=12 ;$ & $\mathrm{b} \oplus=\mathrm{c} ;$ & $\mathrm{c} \boxminus=\mathrm{d} ;$ \\
\hline $\mathrm{d} \ggg=16 ;$ & $\mathrm{d} \oplus=\mathrm{a} ;$ & $\mathrm{a} \boxminus=\mathrm{b} ;$
\end{tabular}

$\operatorname{IQR}(\mathrm{a}, \mathrm{b}, \mathrm{c}, \mathrm{d})$ pseudo code

$\triangleright$ The simplest selection function is defined by

$$
\varphi_{3}\left(b\|c\| \tilde{d}_{i}, k_{b} \| k_{c}\right)=\left(b \boxminus k_{b} \ggg 7\right) \oplus\left(c \boxminus k_{c} \ggg 12\right) \oplus\left(c \boxminus k_{c} \boxminus \tilde{d}_{i}\right)
$$

where $\tilde{d}_{i}=d_{i} \boxminus$ nonce $_{i}$ 


\section{Benefits of the Inverse Quarter Round}

a $\boxplus=b ; \quad d \oplus=a ; \quad d \lll=16 ;$

\begin{tabular}{lll}
\hline $\mathrm{c} \boxplus=\mathrm{d} ;$ & $\mathrm{b} \oplus=\mathrm{c} ;$ & $\mathrm{b} \lll=12 ;$ \\
$\mathrm{a} \boxplus=\mathrm{b} ;$ & $\mathrm{d} \oplus=\mathrm{a} ;$ & $\mathrm{d} \lll=8 ;$ \\
$\mathrm{c} \boxplus=\mathrm{d} ;$ & $\mathrm{b} \oplus=\mathrm{c} ;$ & $\mathrm{b} \lll=7 ;$
\end{tabular}

QR(a,b,c,d) pseudo code

\begin{tabular}{lll}
$\mathrm{b} \ggg=7 ;$ & $\mathrm{b} \oplus=\mathrm{c} ;$ & $\mathrm{c} \boxminus=\mathrm{d} ;$ \\
\hline $\mathrm{d} \ggg=8 ;$ & $\mathrm{d} \oplus=\mathrm{a} ;$ & $\mathrm{a} \boxminus=\mathrm{b} ;$ \\
$\mathrm{b} \ggg=12 ;$ & $\mathrm{b} \oplus=\mathrm{c} ;$ & $\mathrm{c} \boxminus=\mathrm{d} ;$ \\
\hline $\mathrm{d} \ggg=16 ;$ & $\mathrm{d} \oplus=\mathrm{a} ;$ & $\mathrm{a} \boxminus=\mathrm{b} ;$
\end{tabular}

$\operatorname{IQR}(\mathrm{a}, \mathrm{b}, \mathrm{c}, \mathrm{d})$ pseudo code

$\triangleright$ The simplest selection function is defined by

$$
\varphi_{3}\left(b\|c\| \tilde{d}_{i}, k_{b} \| k_{c}\right)=\left(b \boxminus k_{b} \ggg 7\right) \oplus\left(c \boxminus k_{c} \ggg 12\right) \oplus\left(c \boxminus k_{c} \boxminus \tilde{d}_{i}\right)
$$

where $\tilde{d}_{i}=d_{i} \boxminus$ nonce $_{i}$

$\triangleright a$ does not impact the update of $b$ 


\section{Benefits of the Inverse Quarter Round}

a $\boxplus=b ; \quad d \oplus=a ; \quad d \lll=16 ;$

\begin{tabular}{lll}
\hline $\mathrm{c} \boxplus=\mathrm{d} ;$ & $\mathrm{b} \oplus=\mathrm{c} ;$ & $\mathrm{b} \lll=12 ;$ \\
$\mathrm{a} \boxplus=\mathrm{b} ;$ & $\mathrm{d} \oplus=\mathrm{a} ;$ & $\mathrm{d} \lll=8 ;$ \\
$\mathrm{c} \boxplus=\mathrm{d} ;$ & $\mathrm{b} \oplus=\mathrm{c} ;$ & $\mathrm{b} \lll=7 ;$
\end{tabular}

QR(a,b,c,d) pseudo code

\begin{tabular}{lll}
$\mathrm{b} \ggg=7 ;$ & $\mathrm{b} \oplus=\mathrm{c} ;$ & $\mathrm{c} \boxminus=\mathrm{d} ;$ \\
\hline $\mathrm{d} \ggg=8 ;$ & $\mathrm{d} \oplus=\mathrm{a} ;$ & $\mathrm{a} \boxminus=\mathrm{b} ;$ \\
$\mathrm{b} \ggg=12 ;$ & $\mathrm{b} \oplus=\mathrm{c} ;$ & $\mathrm{c} \boxminus=\mathrm{d} ;$ \\
$\mathrm{d} \ggg=16 ;$ & $\mathrm{d} \oplus=\mathrm{a} ;$ & $\mathrm{a} \boxminus=\mathrm{b} ;$
\end{tabular}

$\operatorname{IQR}(\mathrm{a}, \mathrm{b}, \mathrm{c}, \mathrm{d})$ pseudo code

$\triangleright$ The simplest selection function is defined by

$$
\varphi_{3}\left(b\|c\| \tilde{d}_{i}, k_{b} \| k_{c}\right)=\left(b \boxminus k_{b} \ggg 7\right) \oplus\left(c \boxminus k_{c} \ggg 12\right) \oplus\left(c \boxminus k_{c} \boxminus \tilde{d}_{i}\right)
$$

where $\tilde{d}_{i}=d_{i} \boxminus$ nonce $_{i}$

$\triangleright a$ does not impact the update of $b$

$\triangleright$ The probability $p$ of a carry propagation can be estimated

$$
p=\mathbb{P}\left(k_{b}^{[0, x[}>b^{[0, x[}\right)=\frac{2^{x}-\left(b^{[0, x[}+1\right)}{2^{x}}
$$




\section{Benefits of the Inverse Quarter Round}

$\triangleright$ Discarding rotations results in

$$
\varphi_{4, n}\left(b\|c\| \tilde{d}_{i}, k_{b}^{\mathcal{A}}\left\|k_{c}^{\mathcal{B}}\right\| k_{c}^{\mathcal{C}}\right)=\left(b^{\mathcal{A}} \boxminus_{n} k_{b}^{\mathcal{A}}\right) \oplus\left(c^{\mathcal{B}} \boxminus_{n} k_{c}^{\mathcal{B}}\right) \oplus\left(c^{\mathcal{C}} \boxminus_{n} k_{c}^{\mathcal{C}} \boxminus_{n} \tilde{d}_{i}^{\mathcal{C}}\right)
$$

$\triangleright$ Smaller key search space than $\varphi_{2, n}$

$$
|\mathcal{K}|= \begin{cases}2^{3 n}, & \text { if } n \leq 12 \\ 2^{2 n+12}, & \text { if } 12 \leq n \leq 20 \\ 2^{n+32}, & \text { otherwise }\end{cases}
$$

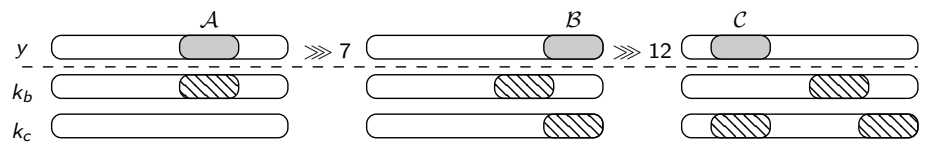

D\&C approach on the ChaCha IQR, $n=8$ 


\section{Benefits of the Inverse Quarter Round}

$D$ Carries were taken into account if $p \geq \frac{3}{4}$

$\triangleright$ Much less collisions than $\varphi_{2, n}$

\section{Proposition}

An attack on $\varphi_{4, n}$ returns 4 collisions.

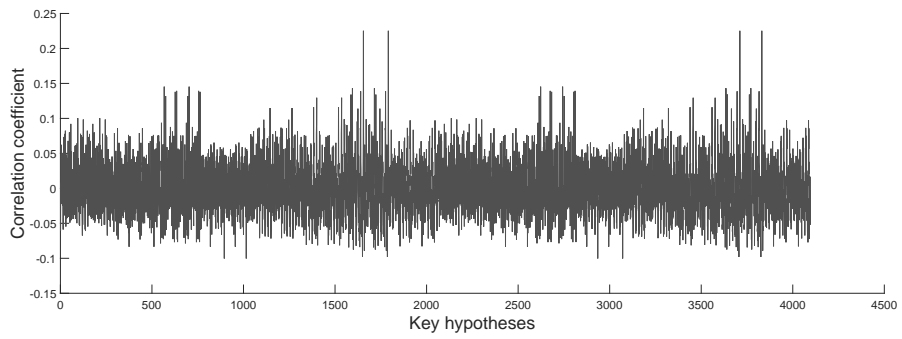

Attack simulation on $\varphi_{4,4}$ 


\section{Bricklayer Attack}

$\triangleright$ Sequential approach

$\triangleright$ Taking advantage of windows previously recovered instead of executing attacks in parallel 


\section{Bricklayer Attack}

$\triangleright$ Sequential approach

$\triangleright$ Taking advantage of windows previously recovered instead of executing attacks in parallel

$\triangleright$ The carry estimation is only necessary during the first attack $\Rightarrow$ especially interesting for $\varphi_{2, n}$ 


\section{Bricklayer Attack}

$\triangleright$ Sequential approach

$\triangleright$ Taking advantage of windows previously recovered instead of executing attacks in parallel

$\triangleright$ The carry estimation is only necessary during the first attack $\Rightarrow$ especially interesting for $\varphi_{2, n}$

$\triangleright$ Collision bits' positions are changed at each attack $\Rightarrow$ some collisions cancelled

$\triangleright$ In the case of $\varphi_{4, n}$, collisions only depends on MSBs $\Rightarrow$ the bricklayer approach allows the correct collision to stand out 


\section{Bricklayer Attack Overview}

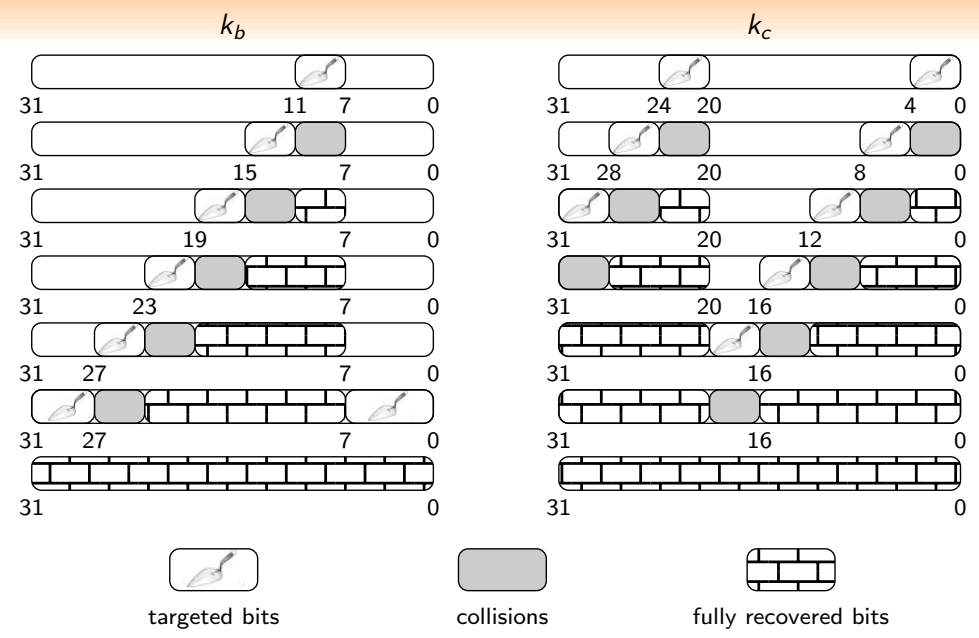

Bricklayer attack example on IQR 


\section{Practical Experiments}
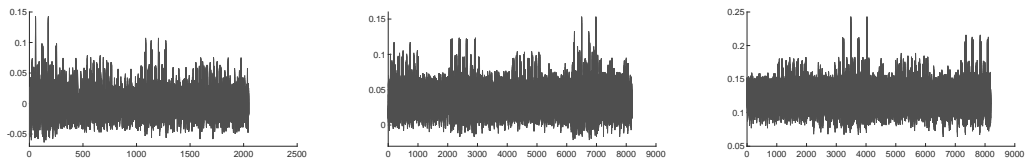

$\kappa=k_{7}^{23 \ldots 20}\left\|k_{7}^{3 \ldots 0}\right\| k_{2}^{10 \ldots 7}$

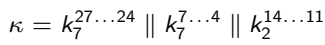

$\kappa=k_{7}^{31 \ldots 28}\left\|k_{7}^{11 \ldots 8}\right\| k_{2}^{18 \ldots 15}$
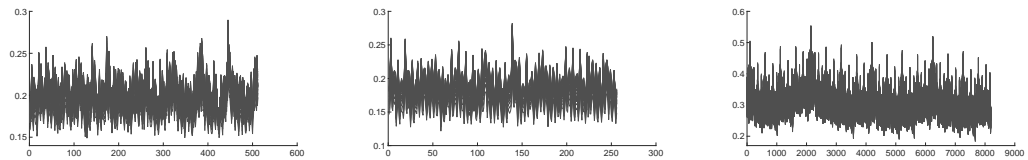

$\kappa=k_{7}^{15 \ldots 12} \| k_{2}^{22 \ldots 19}$

$$
\kappa=k_{7}^{19 \ldots 16} \| k_{2}^{26 \ldots 23}
$$

$$
\kappa=k_{2}^{31 \ldots 27} \| k_{2}^{6 \ldots 0}
$$

Figure: Bricklayer attack against $k_{2}$ and $k_{7}$ 


\section{Application on Existing Protocols}

$\triangleright \varphi_{1}$ requires the knowledge of nonces

$\triangleright \varphi_{3}$ requires the knowledge of plaintexts + ciphertexts + nonces 


\section{Application on Existing Protocols}

$\triangleright \varphi_{1}$ requires the knowledge of nonces

$\triangleright \varphi_{3}$ requires the knowledge of plaintexts + ciphertexts + nonces

$\triangleright$ nonce $_{0, \ldots, 4}=$ counter $\|$ IV 


\section{Application on Existing Protocols}

$\triangleright \varphi_{1}$ requires the knowledge of nonces

$\triangleright \varphi_{3}$ requires the knowledge of plaintexts + ciphertexts + nonces

$\triangleright$ nonce $_{0, \ldots, 4}=$ counter $\|$ IV

$\triangleright$ About TLS

- 96-bit IV is picked randomly for each session

- 32-bit counter is the only predictable part $\Rightarrow 64$ key bits can be recovered at most

- Protocol-level countermeasure 


\section{Application on Existing Protocols}

$\triangleright \varphi_{1}$ requires the knowledge of nonces

$\triangleright \varphi_{3}$ requires the knowledge of plaintexts + ciphertexts + nonces

$\triangleright$ nonce $_{0, \ldots, 4}=$ counter $\|$ IV

$\triangleright$ About TLS

- 96-bit IV is picked randomly for each session

- 32-bit counter is the only predictable part $\Rightarrow 64$ key bits can be recovered at most

- Protocol-level countermeasure

\section{$\triangleright$ About $\mathrm{SSH}$}

- 64-bit IV defined by the packet sequence number 64-bit counter reset for each packet

- Possible to predict the entire nonce! $\Rightarrow$ Need of dedicated countermeasures 


\section{Application on Existing Protocols}

$\triangleright \varphi_{1}$ requires the knowledge of nonces

$\triangleright \varphi_{3}$ requires the knowledge of plaintexts + ciphertexts + nonces

$\triangleright$ nonce $_{0, \ldots, 4}=$ counter $\|$ IV

$\triangleright$ About TLS

- 96-bit IV is picked randomly for each session

- 32-bit counter is the only predictable part $\Rightarrow 64$ key bits can be recovered at most

- Protocol-level countermeasure

\section{$\triangleright$ About $\mathrm{SSH}$}

- 64-bit IV defined by the packet sequence number 64-bit counter reset for each packet

- Possible to predict the entire nonce! $\Rightarrow$ Need of dedicated countermeasures

$\triangleright$ XChaCha construction

Implemented in Libsodium $(\geq 1.0 .12)$

Extend the nonce size to pick it at random

- The nonce is public and must be sent with the cryptogram 


\section{Masking ARX Designs}

$>$ Blinding processed values $x$ using random masks $r \Rightarrow$ impossible to predict intermediate values 


\section{Masking ARX Designs}

$\triangleright$ Blinding processed values $x$ using random masks $r \Rightarrow$ impossible to predict intermediate values

$\triangleright$ ARX designs need both boolean $\left(x^{\prime}=x \oplus r\right)$ and arithmetic $\left(x^{\prime}=x \boxminus r\right)$ masking

$\triangleright$ Two approaches

- Switch from one masking scheme to the other

- Perform additions on the masked values 


\section{Masking ARX Designs}

$\triangleright$ Blinding processed values $x$ using random masks $r \Rightarrow$ impossible to predict intermediate values

$\triangleright$ ARX designs need both boolean $\left(x^{\prime}=x \oplus r\right)$ and arithmetic $\left(x^{\prime}=x \boxminus r\right)$ masking

$\triangleright$ Two approaches

- Switch from one masking scheme to the other

- Perform additions on the masked values

$\triangleright$ Boolean-to-arithmetic conversions are cheap while arithmetic-to-boolean are very heavy

$\triangleright$ Secure adders usually rely on arithmetic to boolean conversions $\Rightarrow$ same complexity

\begin{tabular}{lcc}
\hline & Time & Penalty factor \\
\hline ChaCha20 unmasked & 4380 & 1 \\
ChaCha20 with Karroumi et al. SecAdd [3] & 121618 & 28 \\
ChaCha20 with Coron et al. SecAdd [1] & 93993 & 22 \\
\hline
\end{tabular}

Running time in clock cycles to encrypt a 512-bit block using ChaCha20 on an ARM Cortex-M3 


\section{Conclusion \& Perspectives}

\section{Conlusions}

$\triangleright$ ARX designs remain vulnerable to power/electromagnetic side-channel

$\triangleright$ Our practical setup was able to exploit memory accesses only

$\triangleright$ Introduced the Bricklayer attack with simulated \& practical measurements

$\triangleright$ Harder to attack the QR than its reverse function 


\section{Conclusion \& Perspectives}

\section{Conlusions}

$\triangleright$ ARX designs remain vulnerable to power/electromagnetic side-channel

$\triangleright$ Our practical setup was able to exploit memory accesses only

$\triangleright$ Introduced the Bricklayer attack with simulated \& practical measurements

$\triangleright$ Harder to attack the QR than its reverse function

\section{Open Questions}

$\triangleright$ How could we exploit ALU operations? Is decapping necessary?

$\triangleright$ Can we use these properties to mask a subset of instructions?

$\triangleright$ Is it possible to implement ChaCha20 in a secure way with reasonable performances? 


\section{References}

Jean-Sébastien Coron, Johann Großschädl, Mehdi Tibouchi, and Praveen Kumar Vadnala.

Conversion from Arithmetic to Boolean Masking with Logarithmic Complexity, pages 130-149.

Springer Berlin Heidelberg, Berlin, Heidelberg, 2015.

B. Jungk and S. Bhasin.

Don't fall into a trap: Physical side-channel analysis of ChaCha20-Poly1305.

In Design, Automation Test in Europe Conference Exhibition (DATE), 2017.

Mohamed Karroumi, Benjamin Richard, and Marc Joye.

Addition with Blinded Operands, pages 41-55.

Springer International Publishing, Cham, 2014.

M. Tunstall, N. Hanley, R. McEvoy, C. Whelan, C. Murphy, and W. Marnane.

Correlation Power Analysis of Large Word Sizes. 


\title{
Thank you for your attention!
}

\author{
Questions?
}

